\title{
Associations between social anxiety and emotional intelligence within clinically depressed patients
}

Karen Nolidin ${ }^{1}$, Luke A. Downey ${ }^{1}$, Karen Hansen ${ }^{1}$, Issac Schweitzer ${ }^{2} \&$ Con Stough ${ }^{1}$

${ }^{1}$ Centre for Human Psychopharmacology, Swinburne University of Technology

${ }^{2}$ The Melbourne Clinic, Department of Psychiatry, University of Melbourne

KEYWORDS: Social Anxiety; SAD; Depression; Emotional Intelligence; EI

Address all correspondence to:

Dr Luke Downey

Centre for Human Psychopharmacology

Swinburne University of Technology, Hawthorn,

Victoria 3122

AUSTRALIA

E-mail: 1downey@swin.edu.au

Phone: +61 392145781

Fax: $\quad+61392145230$ 


\begin{abstract}
Background and Objectives: Impairments in Emotional Intelligence (EI) have been found in individuals with high general and social anxiety; however, no studies have examined this relationship in a clinically depressed population. Methods: Thirty-one patients (11 male, 20 female) with a DSM-IV-TR diagnosis of a major affective disorder and 28 non-clinical controls ( 5 male, 23 female) completed self-report instruments assessing EI, depression and social anxiety. Results: Compared to a control group, the clinical group scored lower on the EI dimensions of Emotional Recognition and Expression, Understanding Emotions, Emotional Management, and Emotional Control. Regression analyses revealed Emotional Control was a significant predictor of interaction, performance, and generalised social anxiety. Conclusions: Self-report measures of EI may have predictive value in terms of early identification of those at risk of developing social anxiety and depression. The current study points to the potential value of conducting further studies of a prospective nature.
\end{abstract}




\section{Associations between social anxiety and emotional intelligence within clinically depressed patients}

Social anxiety disorder (SAD) is the most common form of anxiety disorders, and is characterized by a strong fear of social interaction or performance situations because of the potential for embarrassment or humiliation (Jacobs et al., 2008). Social anxiety (or social phobia) is a highly prevalent and co-morbid disorder with depression that is associated with significant burden in terms of its impact on the individual sufferer (Downey et al., 2008), the sufferer's family and society in general in terms of lost productivity (Summerfeldt, Kloosterman, Antony, \& Parker, 2006). The identification of reliable predictors of who is most likely to suffer from SAD could inform the development of prophylactic strategies for protecting individuals prior to SAD onset and development of commonly experienced co-morbidities. The emerging construct of Emotional Intelligence (EI) may constitute such a predictor, and has recently been shown to be related to emotional processing deficits in clinical conditions such as depression (Summerfeldt, Kloosterman, Antony, McCabe, \& Parker, 2011) and generalized anxiety disorder (Dawda \& Hart, 2000).

Broadly defined, EI is considered to be a set of abilities concerned with the regulation, management, control and use of emotions in decision-making, with greater levels of EI being observed to promote healthy and adaptive mental functioning (Baker, Heinrichs, Kim, \& Hofmann, 2002). As such, assessments of EI should intuitively offer a degree of clinical utility within mental health, since the ability of individuals to understand their own emotional states or emotional problems are common indications for a range of clinical conditions (Mattick \& Clarke, 1998). A number of models and measures of EI exist, generally falling into two conceptions of the construct, 'trait' or 
'ability' (Stough, Saklofske, \& Parker, 2009). Currently, two studies have assessed the relationship between social anxiety and EI using the self-report Emotional Quotient Inventory (EQ-I; Bar-On, 1997), one in non-clinical sample (Summerfeldt, et al., 2006), and recently in a clinical sample (Summerfeldt, et al., 2011). Summerfeldt and colleagues observed that better interpersonal and intrapersonal EI abilities were related to lower social interaction anxiety, with EI being the principal predictor of interpersonal adjustment, accounting for the majority of the association with social interaction anxiety within this large non-clinical sample (Summerfeldt, et al., 2006). Similarly, this time utilizing the 'ability' based test, the Mayer-Salovey-Caruso Emotional Intelligence Test (MSCEIT; Mayer et al, 2001), Jacobs and colleagues compared socially phobic individuals with a control group upon the measure of EI and observed that patients with generalized social phobia performed at the normal range for 'ability' EI to the control group. Interestingly, a significant negative association between severity of social anxiety and basic level emotional processing but not higher level strategic emotional processing was observed (Jacobs, et al., 2008). The generalizability of these results is somewhat questionable, given that social anxiety was not assessed within the control group, and as such, the interaction between performance on the EI assessment and anxiety were not assessed individually.

Most recently, the relationship between clinical anxiety and EI has been investigated in samples of social phobia and other anxiety disorders (Summerfeldt, et al., 2011). This study confirmed that intrapersonal EI skills were lower in the sample of social phobia patients than for obsessive compulsive disorder, panic disorder and nonclinical individuals, as well as predicting clinician rated indices of maladjustment. This finding suggests that social phobic individuals ability to recognize, understand and 
express their own emotions is particularly compromised, and this inability is predicts unique variability in maladjustment in comparison to other anxiety disorders. In contrast, in a sample of clinically depressed patients, Downey and colleagues (Downey, et al., 2008) observed significant associations between severity of depression and the EI dimensions of Emotional Management and Emotional Control on the Swinburne University Emotional Intelligence Test (SUEIT: (Palmer \& Stough, 2001)). This finding supported the notion that a lack of emotional control and the inability to regulate emotions are important factors associated with clinical depression, and other similar findings from non-clinical populations (Dawda \& Hart, 2000; Saklofske, Austin, \& Minski, 2003). Given the common co-morbidity of clinical depression and SAD, the current study aimed to investigate the relationship between EI and SAD in a cohort of adults with DSM-IV-TR diagnosis of clinical depression using a cross-sectional sampling strategy.

\section{Method}

\section{Participants}

The clinical sample consisted of 11 male and 20 female patients $(M=51.90$ years, $S D=$ 13.32 years). Twenty-five patients were diagnosed with Major Depression, and six were diagnosed with Bipolar Disorder (three in a depressive phase and three in remission). No patients with a primary diagnosis of SAD were recruited, as social anxiety commonly appears as a co-morbid disorder with Major Depression (Stein \& Kean, 2000). The control group consisted of 28 participants ( 5 male, 23 female) not previously diagnosed with any psychiatric disorder. The mean age of this group was 36.11 years $(S D=14.19$ years $)$ 


\section{Materials}

\section{Liebowitz Social Anxiety Scale (LSAS)}

The LSAS (Liebowitz, 1987) is a 24-item self-report measure of social anxiety that assesses the fear and avoidance aspects of various social situations. The self-report and clinically administered versions correlate highly (Baker, et al., 2002). Respondents must rate the degree to which they fear or feel anxious about certain behaviours and the degree to which they would avoid performing them. Each item requires a rating on a 4point scale from 0 (not at all) to 3 (severe) for fear, and from 0 (never) to 3 (usually) for avoidance. Total scores for the fear and avoidance subscales (range 0-72) are calculated by summing items. Baker and colleagues (Baker, et al., 2002) observed high internal consistencies for this scale (fear: $\propto \propto \neq .89$; avoidance: $\propto \propto=89$ ).

\section{Social Phobia Scale (SPS) and Social Interaction Anxiety Scale (SIAS)}

The 20-item SPS and 19-item SIAS were created concurrently by Mattick and Clark (Mattick \& Clarke, 1998). Respondents must rate the degree to which each statement characterizes them on a 5-point scale from 0 (not at all) to 4 (extremely). The score range is 0-80 for the SPS and 0-76 for the SIAS. Mattick and Clark (1998) found high internal consistencies for both scales $(\alpha=.88-.94)$ in different sample groups (i.e., undergraduate students; a community sample; and patients with SAD, agoraphobia, and simple phobia). Although these social anxiety measures overlap, each assesses different aspects: the LSAS measures generalised social anxiety (anxiety about performance and interaction situations); the SPS measures performance anxiety (anxiety about being observed whilst performing certain activities); and the SIAS measures interaction anxiety (anxiety about generally interacting with people socially). 
Swinburne University Emotional Intelligence Test (SUEIT)

The SUEIT (Palmer \& Stough, 2001) is a 64-item self-report EI measure. Respondents must rate the degree that each statement characterizes them on a scale from 1 (never) to 5 (always). Five separate scores can be calculated for the EI dimensions of Emotional Recognition and Expression (ERE), Understanding others' Emotions (UE), Emotions Direct Cognition (EDC), Emotional Management (EM), and Emotional Control (EC); plus a total EI score (Downey, Papageorgiou, \& Stough, 2006). Palmer and Stough (2001) found reasonable reliability in a general population $(\alpha \geq .72$ for total EI and each dimension). The SUEIT has been used in a clinically depressed sample previously (Downey, et al., 2008).

The Beck Depression Inventory $-2^{\text {nd }}$ Edition (BDI-II)

The BDI-II (Beck, Steer, \& Brown, 1996) is a 21-item self-report scale for measuring severity of depression. Respondents must rate the degree to which they are experiencing depressive symptoms on a 4-point scale from 0 (not at all) to 3 (severely). Scores range from 0 to 84 and are calculated by summing responses. The BDI-II has demonstrated excellent test-retest reliabilty, internal consistency, convergent and discriminant validity (Beck, et al., 1996).

\section{Beck Anxiety Inventory (BAI)}

The BAI (Beck \& Steer, 1993) is a 21-item self-report scale that measures anxiety severity. Respondents must rate the degree to which they are experiencing general anxiety symptoms on a 4-point scale from 0 (not at all) to 3 (severely). Scores range 
from 0 to 84 and are obtained by summing responses. This scale has demonstrated excellent test-retest correlations, internal consistency, and convergent and discriminant validity (Beck \& Steer, 1993).

\section{Procedure}

Patients were recruited from the Professorial Unit of the Melbourne Clinic, a private psychiatric facility in Melbourne, Australia, that receives referrals from throughout Victoria. All patients were first screened for suitability by their treating clinician and eligible patients were approached and informed about the project. Participants from the control group were recruited through word-of-mouth. Individuals gave their consent to participate by signing an informed consent form and the study was approved by both the Melbourne Clinic and Swinburne University Human Research Ethics Committees.

\section{Results}

Means and standard deviations of the social anxiety, depression, general anxiety, and EI scores for the clinical and control groups are displayed in Table 1. One-way analyses of variance (ANOVA) were used to compare depression severity and general anxiety scores between the two groups. Patients predictably scored higher than the controls on the measures of depression severity $\left(F_{(1,57)}=35.95, p<.001\right)$ and general anxiety $\left(F_{(1,57)}\right.$ $=27.65, p<.001)$. 
Insert Table 1 here

A multivariate analysis of variance (MANOVA) was used to compare SUEIT dimensions scores of the clinical and control groups. An overall multivariate effect was found (Wilks' $\lambda=.688, F_{(5,53)}=4.81, p<.01$, partial $\eta^{2}=.31$ ). Univariate $F$-tests revealed clinical patients scored lower than the control participants on $\operatorname{ERE}\left(F_{(1,57)}=\right.$ $13.14, p<.01$, partial $\left.\eta^{2}=.19\right), \operatorname{UE}\left(F_{(1,57)}=8.78, p<.01\right.$, partial $\left.\eta^{2}=.13\right), \operatorname{EM}\left(F_{(1,57)}=\right.$ $20.63, p<.001$, partial $\left.\eta^{2}=.27\right)$, and $\mathrm{EC}\left(F_{(1,57)}=13.75, p<.001\right.$, partial $\left.\eta^{2}=.19\right)$. There was no significant difference for $\operatorname{EDC}\left(F_{(1,57)}=.24, p=.624\right.$, partial $\left.\eta^{2}=.004\right)$. These analyses indicate that the clinically depressed group scored lower on 4 of the 5 dimensions of EI from the SUEIT consistent with previous work with clinically depressed patients (Downey, et al., 2008). A MANOVA was performed to compare the clinical and control groups on measures of social anxiety. An overall multivariate effect was found (Wilks' $\lambda=.736, F_{(4,52)}=4.67, p<.005$, partial $\left.\eta^{2}=.26\right)$. Univariate $F$-tests revealed clinical patients scored significantly higher than control participants on the LSAS fear subscale $\left(F_{(1,55)}=13.97, p<.001\right.$, partial $\left.\eta^{2}=.20\right)$, the SIAS $\left(F_{(1,55)}=13.77, p\right.$ $<.001$, partial $\left.\eta^{2}=.20\right)$, and the SPS $\left(F_{(1,55)}=19.43, p<.001\right.$, partial $\left.\eta^{2}=.26\right)$. There were no significant difference on the LSAS avoidance subscale $\left(F_{(1,55)}=1.99, p=.164\right.$, partial $\left.\eta^{2}=.035\right)$.

To determine the relative contribution of EI to the severity of social anxiety, a series of linear regression analyses were performed with the EI dimensions as the independent variables, and the social anxiety scores as the dependent variables. 
Correlational analyses (Table 2) revealed the LSAS avoidance subscale did not significantly correlate with the EI dimensions, so it was not analysed.

Insert Table 2 here

For the first analysis, a simple linear regression analysis was performed with LSAS fear as the dependent variable. The EI dimensions with significant correlations with LSAS fear (ERE, EM, and EC) were included as predictors in the model. The direct model was significant $\left(\mathrm{F}_{3,26}=7.35, \mathrm{P}<.001, \mathrm{R}=.68, \mathrm{R}^{2}=.46\right)$. However, $\mathrm{EC}$ was the only significant predictor $(\mathrm{p}=.01)$. For the second analysis, linear regression was performed with SIAS as the dependent variable. The EI dimensions with significant correlations with SIAS (ERE, EM, and EC) were included as predictors in the model. The direct model was significant $\left(\mathrm{F}_{3,27}=10.05, \mathrm{P}<.001, \mathrm{R}=.73, \mathrm{R}^{2}=.53\right)$ and again $\mathrm{EC}$ was the only significant predictor $(\mathrm{p}<.01)$. For the third analysis, a simple linear regression analysis was performed with SPS was the dependent variable. The EI dimensions with significant correlations with SPS (EM and EC) were included as predictors in the model. The direct model was significant $\left(\mathrm{F}_{2,28}=10.69, \mathrm{P}<.001, \mathrm{R}=.66, \mathrm{R}^{2}=.43\right)$ and $\mathrm{EC}$ was a significant predictor $(\mathrm{p}<.01)$. These results taken together suggest that Emotional Control is a significant predictor of LSAS fear, SIAS and SPS. In order to examine whether EC still predicted the measures of social anxiety after controlling for depression and general anxiety we recalculated the regressions with depression and general anxiety included. After controlling for depression and general anxiety, EC $(\mathrm{p}<.05)$ was only able to predict interaction anxiety $\left(\mathrm{F}_{5,25}=11.3, \mathrm{P}<.001, \mathrm{R}=.83, \mathrm{R}^{2}=.69\right)$.

\section{Discussion}


The present study examined the association between a self-report measure of EI and social anxiety in a clinical sample of depressed adults. Compared to the control group, the clinically depressed patients scored lower on the EI dimensions ERE, UE, EM and EC but not on the EDC subscale, which is more related to the use of emotions in guiding decision making. This pattern of results confirms the findings of a previous study concerning deficits in the ability to recognise and express emotions, manage positive and negative emotions adaptively and control strong emotions in a clinically depressed sample (Downey, et al., 2008) and that the ability to manage and control emotions is related to severity of depression. More specific analyses indicated that EC significantly predicted interaction, performance, and generalised social anxiety, with poorer control of strong negative emotions predicting greater social anxiety. The clinical group also reported greater social anxiety than the control group which was expected due to the diagnoses of these patients. Given social and general anxiety are often observed in patients with clinical depression and the prevalence of this disorder (Issakidis, Sanderson, Corry, Andrews, \& Lapsley, 2004), research concerning the various linkages between emotion processing ability, anxiety and depression is required (Summerfeldt, et al., 2011).

The results suggest that in terms of emotional processing, individuals with depression and co-morbidly experiencing social anxiety are poorer at recognising, describing, perceiving others' emotions and managing and controlling emotions than controls. Control of strong emotions predicted generalised social anxiety, interaction anxiety, and performance anxiety. After controlling for depression and general anxiety this dimension predicted interaction anxiety - high interaction anxiety was explained by having poor control of strong emotions. Therefore, in terms of severity of symptoms, EC 
was the dimension of EI that was most predictive. The inability to tolerate strong emotions and to operate effectively under threat of these strong emotions is what determines the severity of the patient's social anxiety symptoms (Beck \& Steer, 1993). These results may have implications for interventions such as cognitive behavioural therapy. A patient with low EC and high social anxiety may need to first learn how to tolerate the emotions and then address the distorted cognitions underlying them. As the present study is one of the few to investigate EI and social anxiety in a clinical sample, future research is required to replicate and extend the study especially given the inconsistencies within previous studies methodologies and findings and the purported utility of EI development programs (Hansen, Gardner, \& Stough, 2007).

Our clinical group was observed scored higher than the Summerfeldt and colleagues study sample (Summerfeldt, et al., 2006) upon the same social anxiety measures, but close to the group norms for the SIAS (Mattick \& Clarke, 1998) and LSAS fear subscale (Baker, et al., 2002). Unexpectedly the LSAS avoidance subscale did not significantly correlate with any of the dimensions of EI, possibly due to the lack of a primary diagnoses with SAD. It was also surprising ERE, UE, and EM did not predict social anxiety, particularly given Summerfeldt and colleagues have reported comparable 'intrapersonal' dimensions of EI were predictive of social anxiety and social phobia (Summerfeldt, et al., 2011; Summerfeldt, et al., 2006). Again this may be due to differences in severity of social anxiety seen across the two different samples (clinical versus non-clinical), or the relationship between social anxiety and EI may be complex or mediated by other factors including co-morbidly experienced clinical disorders. Our results also differ somewhat from a similar study conducted by Jacobs et al. (2008) 
found no significant differences in ability EI between patients and matched controls, possibly due to differences in the EI assessments. The 'ability' measure utilised by Jacobs et al (2008) reflect 'maximal performance' scores rather than how the patients are typically processing emotional information (Downey, Lee, \& Stough, 2011). As such, results from self-reported measures may provide more ecologically valid assessment of the episodic effects of depression and SAD in clinical populations. Whether deficits in self-reported EI are also sensitive to a vulnerability to depressive of social anxiety symptoms would need to be examined in a fully prospective study.

Future research should examine the utility of EI development programs for patients with clinical disorders as they may offer an effective, innovative and robust method to improve emotional competencies, particularly when interaction anxiety is concerned. Given reports that high levels of EI and in particular the control and management of emotions leads to improved mental health and life satisfaction (Schutte, Malouff, Thorsteinsson, Bhullar, \& Rooke, 2007) the assessment of EI in individuals at-risk for depression and social anxiety should be a priority. 
Baker, S. L., Heinrichs, N., Kim, H. J., \& Hofmann, S. G. (2002). The Liebowitz Social Anxiety Scale as a self-report instrument: a preliminary psychometric analysis. Behaviour Research and Therapy, 40(6), 701-715.

Beck, A. T., \& Steer, R. A. (1993). BAI Manual. San Antonio: Harcourt Brace \& Company.

Beck, A. T., Steer, R. A., \& Brown, G. K. (1996). BDI-II Manual. San Antonio: Harcourt Brace \& Company.

Dawda, D., \& Hart, S. D. (2000). Assessing emotional intelligence: Reliability and validity of the Bar-On Emotional Quotient Inventory (EQ-i) in university students. Personality and Individual Differences, 28(4), 797-812.

Downey, L. A., Johnston, P. J., Hansen, K., Schembri, R., Stough, C., Tuckwell, V., et al. (2008). The relationship between emotional intelligence and depression in a clinical sample. European Journal of Psychiatry, 22(2), 93-98.

Downey, L. A., Lee, B., \& Stough, C. (2011). Recruitment consultant revenue: Relationships with IQ, personality, and emotional intelligence. International Journal of Selection and Assessment, 19(3), 280-286. doi: 10.1111/j.14682389.2011.00557.x

Downey, L. A., Papageorgiou, V., \& Stough, C. (2006). Examining the relationship between leadership, emotional intelligence and intuition in senior female managers. Leadership and Organization Development Journal, 27(4), 250-264. doi: 10.1108/01437730610666019

Hansen, K., Gardner, L., \& Stough, C. (2007). Improving occupational stress through emotional intelligence development. Organisations and People, 14(2), 70.

Issakidis, C., Sanderson, K., Corry, J., Andrews, G., \& Lapsley, H. (2004). Modelling the population cost-effectiveness of current and evidence-based optimal treatment for anxiety disorders. Psychological Medicine, 34(1), 19-35. doi: $10.1017 / \mathrm{s} 003329170300881 \mathrm{x}$

Jacobs, M., Snow, J., Geraci, M., Vythilingam, M., Blair, R. J. R., Charney, D. S., et al. (2008). Association between level of emotional intelligence and severity of anxiety in generalized social phobia. Journal of Anxiety Disorders, 22(8), 14871495. doi: 10.1016/j.janxdis.2008.03.003

Liebowitz, M. R. (1987). Social phobia. Modern problems of pharmacopsychiatry, 22, 141-173.

Mattick, R. P., \& Clarke, J. C. (1998). Development and validation of measures of social phobia scrutiny fear and social interaction anxiety1. Behaviour Research and Therapy, 36(4), 455-470.

Palmer, B., \& Stough, C. (2001). Workplace SUEIT: Swinburne University Emotional Intelligence Test - Descriptive Report: Organisational Psychology Research Unit, Swinburne University.

Saklofske, D. H., Austin, E. J., \& Minski, P. S. (2003). Factor structure and validity of a trait emotional intelligence measure. Personality and Individual Differences, 34(4), 707-721. doi: 10.1016/s0191-8869(02)00056-9

Schutte, N. S., Malouff, J. M., Thorsteinsson, E. B., Bhullar, N., \& Rooke, S. E. (2007). A meta-analytic investigation of the relationship between emotional intelligence 
and health. Personality and Individual Differences, 42(6), 921-933. doi: 10.1016/j.paid.2006.09.003

Stein, M. B., \& Kean, Y. M. (2000). Disability and quality of life in social phobia: epidemiologic findings. American Journal of Psychiatry, 157(10), 1606-1613.

Stough, C., Saklofske, D. H., \& Parker, J. D. A. (2009). A Brief Analysis of 20 Years of Emotional Intelligence: An Introduction to Current Advances in EI Assessment and Application: Springer.

Summerfeldt, L. J., Kloosterman, P. H., Antony, M. M., McCabe, R. E., \& Parker, J. D. A. (2011). Emotional intelligence in social phobia and other anxiety disorders. Journal of Psychopathology and Behavioral Assessment, 33(1), 69-78. doi: 10.1007/s10862-010-9199-0

Summerfeldt, L. J., Kloosterman, P. H., Antony, M. M., \& Parker, J. D. A. (2006). Social anxiety, emotional intelligence, and interpersonal adjustment. Journal of Psychopathology and Behavioral Assessment, 28(1), 57-68. doi: 10.1007/s10862-006-4542-1 
Table 1: Means and Standard Deviations of the Clinical and Control Groups

\begin{tabular}{|c|c|c|c|c|c|c|}
\hline & \multicolumn{3}{|c|}{ Clinical } & \multicolumn{3}{|c|}{ Control } \\
\hline & $M$ & $S D$ & $n$ & $M$ & $S D$ & $n$ \\
\hline LSAS fear & 36.73 & 19.17 & 30 & 20.15 & 13.49 & 27 \\
\hline LSAS avoidance & 27.50 & 17.21 & 30 & 21.81 & 12.57 & 27 \\
\hline Interaction Anxiety & 37.33 & 20.32 & 30 & 20.04 & 13.87 & 27 \\
\hline Performance Anxiety & 28.23 & 21.56 & 30 & 8.70 & 8.47 & 27 \\
\hline Emotional Recognition and Expression & 31.19 & 7.29 & 31 & 37.61 & 6.18 & 28 \\
\hline Understanding Emotions & 62.61 & 8.85 & 31 & 69.61 & 9.28 & 28 \\
\hline Emotions Direct Cognition & 34.06 & 5.27 & 31 & 34.79 & 5.95 & 28 \\
\hline Emotional Management & 28.55 & 8.32 & 31 & 36.82 & 5.11 & 28 \\
\hline Emotional Control & 22.52 & 7.33 & 31 & 28.25 & 3.82 & 28 \\
\hline Total EI & 178.94 & 25.37 & 31 & 207.07 & 22.30 & 28 \\
\hline Depression & 27.55 & 16.18 & 31 & 7.54 & 5.00 & 28 \\
\hline Anxiety & 18.42 & 12.08 & 31 & 7.42 & 6.33 & 28 \\
\hline
\end{tabular}

LSAS: Liebowitz Social Anxiety Scale; EI: Emotional Intelligence 
Table 2: Intercorrelations Among the Variables for Clinical Group

\begin{tabular}{|c|c|c|c|c|c|c|c|c|c|c|c|c|}
\hline & ERE & UE & EDC & EM & $\mathrm{EC}$ & $\begin{array}{c}\text { Total } \\
\text { EI }\end{array}$ & $\begin{array}{c}\text { LSAS } \\
\text { fear }\end{array}$ & $\begin{array}{l}\text { LSAS } \\
\text { avoid }\end{array}$ & SIAS & SPS & BDI-II & BAI \\
\hline \multicolumn{13}{|l|}{ ERE } \\
\hline UE & $.54 * *$ & . & & & & & & & & & & \\
\hline EDC & .12 & .31 & . & & & & & & & & & \\
\hline EM & $.52 * *$ & .25 & .20 & . & & & & & & & & \\
\hline $\mathrm{EC}$ & $.44^{*}$ & -.03 & -.06 & $.80 * *$ & . & & & & & & & \\
\hline Total EI & $.80 * *$ & $.64 * *$ & $.40 *$ & $.84 * *$ & $.65^{* *}$ & . & & & & & & \\
\hline $\begin{array}{l}\text { LSAS } \\
\text { fear }\end{array}$ & $-.40 *$ & .09 & .17 & $-.56^{* *}$ & $-.67 * *$ & $-.42 *$ & & & & & & \\
\hline $\begin{array}{l}\text { LSAS } \\
\text { avoid }\end{array}$ & -.04 & -.11 & .13 & -.14 & -.16 & -.12 & $.49 * *$ & . & & & & \\
\hline SIAS & $-.47 * *$ & .08 & .30 & $-.55^{* *}$ & $-.70^{* *}$ & $-.42^{*}$ & $.90 * *$ & .34 & . & & & \\
\hline SPS & -.28 & .24 & .25 & $-.52 * *$ & $-.66^{* *}$ & -.31 & $.83 * *$ & .23 & $.87 * *$ & . & & \\
\hline BDI-II & $-.48 * *$ & -.06 & .10 & $-.71 * *$ & $-.69 * *$ & $-.57 * *$ & $.63 * *$ & .09 & $.74 * *$ & $.71 * *$ & . & \\
\hline BAI & $-.44 *$ & -.05 & .11 & $-.60 * *$ & $-.67 * *$ & $-.51 * *$ & $.74 * *$ & .31 & $.74 * *$ & $.80 * *$ & $.72 * *$ & . \\
\hline
\end{tabular}

ERE: Emotional Recognition and Expression; UE: Understanding other's Emotions; EDC: Emotions Direct Cognition; EM:

Emotional Management; EC: Emotional Control; EI: Emotional Intelligence LSAS: Liebowitz Social Anxiety Scale; SIAS:

Social Interaction Anxiety Scale; SPS: Social Phobia Scale; BDI-II: Beck Depression Inventory; BAI: Beck Anxiety Inventory Note: $N=31$, except LSAS fear and LSAS perf $N=30 ; * *=\mathrm{p}<.01 ; *=p<.05$ 
Social Anxiety and Emotional Intelligence 
Dr Luke Downey's research experience and expertise is in the area of human neurocognitive assessment and neuropsychopharmacology of human cognition and behaviour. He is an active member of The Centre for Human Psychopharmacology, which involves the development of cognitive testing batteries for a variety of illicit substances, natural medicines and herbal extracts. Dr Downey gained considerable experience conducting and managing large-scale clinical trials of illicit interventions in human volunteers, as well as knowledge of GCP procedures and contractual/IP issues related to industry and other organisational partnerships. Further to this, he has a comprehensive experience in the design, development and implementation of rigorous research methods for the assessment of pharmacology-cognition and nutrition-cognition associations. Dr Downey is currently undertaking a NH\&MRC fellowship (CJ Martin Overseas Biomedical Fellowship) in conjunction with Professor Andy Parrott at Swansea University looking into the health effects of acute and cumulative use of Esctasy/MDMA. 
K. Nolidin, is currently a PhD student at The Centre for Human Psychopharmacology, Swinburne University.

L. A. Downey, PhD, is a NH\&MRC Early Career Fellow (CJ Martin Overseas Biomedical Fellow) working at The Centre for Human Psychopharmacology, Swinburne University, Australia and Department of Psychology, College of Human and Health Sciences, Swansea University, UK.

$\mathrm{K}$. Hansen, $\mathrm{PhD}$, is a postdoctoral researcher at The Centre for Human Psychopharmacology, Swinburne University.

I. Schweitzer, Professor Isaac Schweitzer was an international expert in the treatment of the mood disorders, major depression and bipolar disorder. He investigated mood disorders extensively, including the biological and psychosocial aspects of these conditions. His research has covered neuroendocrine, neuroimaging, genetic, and pharmacological studies. He passed away on the 1-2-2013.

C. Stough, PhD, is Professor of Cognitive Neuroscience and Psychology at Swinburne University of Technology, Australia, director of the Centre for Human Psychopharmacology. 